\title{
Données médicales: quelle dose de transparence, à l'avenir?
}

\author{
Jacques de Haller \\ Président du Comité forumsante.ch
}

\author{
Dans la série des questions "politiquement incorrectes", en politique de santé, \\ figure actuellement en bonne place celle des limites du secret médical: doit-il \\ vraiment être absolu, face à l'accumulation des banques de données et aux possi- \\ bilités qu'ouvre l'analyse de ces données?
}

Le secret médical fait partie des mythes fondateurs de la médecine; c'est, en politique de santé, l'un de ces points de "douleur exquise», comme on dit en clinique, qui cumule tabou et passion. Mais le secret médical apporte en réalité avec lui pas mal d'ambiguïté - respectable et vénéré, indispensable, il est parfois instrumentalisé à l'encontre de ceux-là même qu'il devrait servir; et c'est aussi une arme politique redoutablement efficace, on l'a vu plusieurs fois. Et puis, à propos de données médicales, la transparence ellemême est équivoque: souhaitée pour les informations et leur stockage, elle est vigoureusement rejetée lorsqu'elle s'applique au patient!

Difficile donc de discuter sereinement du secret médical et de la transparence. Toucher au secret, c'est toucher au sacré...

Reste qu'il faudra bien se mettre à réfléchir, car si l'on considère la situation avec un soupçon d'objectivité, la nécessité d'une réflexion renouvelée semble évidente. Oui, cela fait 2500 ans qu'Hippocrate a posé, avec le secret médical, l'une des bases fondamentales de la confiance entre médecin et patient - base aussi, du coup, de la relation thérapeutique et de la qualité du traitement. Mais quand les informations s'accumulent comme elles le font actuellement, que leur côté secret est souvent une fiction, que leur «cache» n'est pas inviolable, et que leur utilisation raisonnée profiterait à toutes et tous, individuellement et collectivement, on devrait pouvoir imaginer briser quelques tabous. Que ce soit dans les dossiers des médecins ou des hôpi-

Le mardi 3 février 2015 à l'hôtel Bellevue à Berne, forumsante.ch organise pour la $16^{\mathrm{e}}$ fois une journée de réflexion sur un thème d'actualité: «Transparence des données médicales: à quelle dose?». Des conférenciers suisses et internationaux de haut niveau traiteront des aspects éthiques, juridiques, politiques, et bien sûr médicaux, de cette question. forumsante.ch ne s'arrête pas à ce qui est, mais s'attache traditionnellement à envisager l'avenir - on y expose, construit et échange des idées pointues et originales sur le futur. Site Internet (programme): www.forumsante.ch

Inscriptions: forumsante[at]furrerhugi.ch taux, dans les bureaux des assureurs ou dans les ordinateurs des chercheurs de l'industrie, les données médicales des patient-e-s existent en quantités immenses; elle sont souvent tenues secrètes par principe, ce qui n'est pas mauvais en soi, mais elles ne peuvent du coup pas être mises à profit comme elles pourraient l'être dans des champs du système de santé où elles sont irremplaçables: pour la prévention, pour la promotion et le contrôle de la qualité, pour la santé publique, sans parler de la gestion du système de santé lui-même. Ce sont des domaines où l'anonymisation n'est pas toujours possible ou souhaitable dans la médecine personnalisée, dans la promotion de la qualité et la prévention chez les malades chroniques dans le cadre des modèles de soins, voire dans l'amélioration de la gestion du système par la transparence. De nombreuses questions se posent donc si l'on se confronte sans préjugé à la question du secret médical des questions qui deviennent pressantes et auxquelles le système de santé se devrait d'apporter réponse:

- De quelle manière et dans quelles limites le secret médical peut-il être assoupli, au vu du développement technologique et de l'évolution de la société?

- Quelles sont les valeurs, derrière le concept actuel de secret médical, qui doivent être préservées? Lesquelles sont périmées?

- Que pourrait-on faire avec les données existantes, que le concept actuel de secret médical empêche d'exploiter?

- Quels avantages le patient peut-il retirer de l'assouplissement du secret médical? Et les autres acteurs du monde de la santé?

On doit souhaiter à chacun des partenaires de notre système de santé qu'il ose s'attaquer à ces questions, dans le respect des besoins de chacun, avant d'être dépassé par l'évolution tant de la société que de la technologie. 